\title{
Aerosol-cirrus interactions: a number based phenomenon at all?
}

\author{
M. Seifert ${ }^{1,2}$, J. Ström² ${ }^{2}$ R. Krejci ${ }^{1,2}$, A. Minikin ${ }^{3}$, A. Petzold ${ }^{3}$, J.-F. Gayet ${ }^{4}$, H. Schlager ${ }^{3}$, H. Ziereis ${ }^{3}$, U. Schumann ${ }^{3}$, \\ and J. Ovarlez ${ }^{5}$ \\ ${ }^{1}$ Department of Meteorology, Stockholm University, Stockholm, Sweden \\ ${ }^{2}$ Air Pollution Laboratory, Institute for Applied Environmental Research, Stockholm University, Stockholm, Sweden \\ ${ }^{3}$ Deutsches Zentrum für Luft- und Raumfahrt, Institut für Physik der Atmosphäre, Oberpfaffenhofen, Germany \\ ${ }^{4}$ Laboratoire de Météorologie Physique, Université Blaise Pascal, Clermont-Ferrand, France \\ ${ }^{5}$ Laboratoire de Météorologie Dynamique, Ecole Polytechnique, Palaiseau, France
}

Received: 5 May 2003 - Published in Atmos. Chem. Phys. Discuss.: 15 July 2003

Revised: 17 November 2003 - Accepted: 7 February 2004 - Published: 13 February 2004

\begin{abstract}
In situ measurements of the partitioning of aerosol particles within cirrus clouds were used to investigate aerosol-cloud interactions in ice clouds. The number density of interstitial aerosol particles (non-activated particles in between the cirrus crystals) was compared to the number density of cirrus crystal residuals. The data was obtained during the two INCA (Interhemispheric Differences in Cirrus Properties from Anthropogenic Emissions) campaigns, performed in the Southern Hemisphere (SH) and Northern Hemisphere (NH) midlatitudes. Different aerosol-cirrus interactions can be linked to the different stages of the cirrus lifecycle. Cloud formation is linked to positive correlations between the number density of interstitial aerosol (Nint) and crystal residuals (Ncvi), whereas the correlations are smaller or even negative in a dissolving cloud. Unlike warm clouds, where the number density of cloud droplets is positively related to the aerosol number density, we observed a rather complex relationship when expressing Ncvi as a function of Nint for forming clouds. The data sets are similar in that they both show local maxima in the Nint range 100 to $200 \mathrm{~cm}^{-3}$, where the SH- maximum is shifted towards the higher value. For lower number densities Nint and Ncvi are positively related. The slopes emerging from the data suggest that a tenfold increase in the aerosol number density corresponds to a 3 to 4 times increase in the crystal number density. As Nint increases beyond the ca. 100 to $200 \mathrm{~cm}^{-3}$, the mean crystal number density decreases at about the same rate for both data sets. For much higher aerosol number densities, only present in the NH data set, the mean Ncvi remains low. The situation for dissolving clouds allows us to offer two possible, but at this point only speculative, alternative interactions between aerosols and cirrus: evaporating clouds might be associated with a source of aerosol particles, or air pollution (high aerosol number density) might retard ice particle evaporation rates.
\end{abstract}

Correspondence to: $\mathrm{M}$. Seifert

(marco.seifert@itm.su.se)

\section{Introduction}

Recent climate assessments have emphasized the importance of anthropogenic changes in aerosol loading and its potential impact on climate by influencing cloud microphysics and thereby changes in cloud reflectivity (Haywood and Boucher, 2000; IPPC 2001), which is referred to as the indirect aerosol effect.

Aerosol particles play an important role in the formation of clouds since they reduce the supersaturation threshold for cloud formation, which would be very high in an atmosphere without particles. The so-called Twomey effect, of enhanced cloud droplet number density in warm clouds, as a result of an increase in cloud condensation nuclei, has been discussed in many studies (e.g. Twomey, 1974; Cohard et al., 1998). Changes in the cloud droplet spectrum are not only affecting cloud albedo, but are thought to influence cloud lifetimes and the hydrological cycle in the atmosphere as well. The Twomey effect has been confirmed in various field studies (e.g. Noone et al., 2000; Ackerman et al., 2000).

The role of aerosols in the formation of cold clouds is still an open issue and very little data is available to compare with what is expected from numerical simulations. The relation between aerosol and cloud elements is somewhat more complex in ice clouds than in warm clouds due to the different modes of nucleation: homogeneous and heterogeneous nucleation. The relative importance of homogeneous and heterogeneous nucleation during cloud formation is still a matter of debate. Which nucleation mode is thought to dominate under atmospheric conditions is critical to the estimates on the aerosol induced anthropogenic impact on cirrus clouds. DeMott et al. (1994) suggested that changing aerosol size and composition may have a large impact on cirrus formation and the crystal number density. Other studies showed the opposite and concluded that aerosol properties do not significantly affect the microphysical and radiative properties of cirrus clouds (e.g. Jensen and Toon, 1994). Numerical 
simulations using freezing rates based on recent laboratory measurements suggest that the effect from changes in ambient sulfate aerosol properties may have a marginal effect on the microphysical properties and lifecycle of cirrus (Kärcher and Lohmann, 2002; Lohmann and Kärcher, 2002). However, if at least two types of freezing aerosol particles compete during cloud formation, adding efficient ice nuclei to liquid sulfate aerosols can lead to a marked suppression of pristine ice crystal number densities (Kärcher and Lohmann, 2003). Using a large number of numerical simulations driven by observed distributions of vertical wind and aerosol size distributions, Kärcher and Ström (2003) emphasized that it may be difficult to assess the relative importance of the indirect aerosol effect and changes in the dynamical forcing patterns on cirrus clouds in a future climate. Both factors could alter cirrus cloud properties by similar amounts. Observations of an aerosol-induced change in cirrus properties are few. In situ observations within, and in the vicinity of, air traffic corridors show a possible link between aircraft emissions and cirrus microphysical properties (Kristensson et al., 2000; Ström and Ohlsson, 1998). However, a more general approach to study the aerosol cirrus interaction, and to test the sensitivity of cirrus to changes in ambient aerosol using in situ data, has not been previously applied to the authors' knowledge.

The main goal of this study is to present a first attempt to improve our understanding of cloud- aerosol interactions based on the largest data set presently available of concurrent detailed aerosol and cirrus cloud microphysical data. This includes the investigation of the aerosol induced change in the crystal number density as well as the investigation of a possible cloud induced change in the aerosol number density. The measurements presented in this study were performed during the INCA project (Interhemispheric differences in cirrus properties from anthropogenic emissions). As the name indicates, the project was designed to compare cirrus properties in clean and polluted air masses. The two campaigns, one in the Southern Hemisphere (SH) and one in the Northern Hemisphere (NH) midlatitudes, provided data from two very different environments covering a wide range of aerosol number densities and air masses. The analysis will focus on the similarities and differences in the relationship between the number density of crystals and aerosol particles observed in the two campaigns.

\section{Experimental data}

Two aircraft campaigns were performed using the research aircraft Falcon, operated by Deutsches Zentrum fr Luft- und Raumfahrt. The measurements were conducted using an identical payload in comparable meteorological conditions during equivalent seasons, within the same year. The $\mathrm{SH}$ campaign took place in March/April 2000 (Punta Arenas, Chile, $54^{\circ} \mathrm{S}$ ) and the $\mathrm{NH}$ campaign in September/November
2000 (Prestwick, Scotland, 53 ${ }^{\circ}$ N). For more information on the project we refer to http:/www.pa.op.dlr.de/inca/.

\subsection{Instrumentation}

Trace gas measurements provided information on the origin of the observed air masses. The most important tracer concerning cirrus clouds is water vapor, which was measured by a frost-point hygrometer (Ovarlez et al., 2000). From the primary measurement of the frost point we derived the relative humidity over ice (RHi) with a $2 \sigma$ relative uncertainty better than $7 \%$. In addition we made use of $\mathrm{CO}$ to identify recent transport of polluted air from the boundary layer and $\mathrm{O}_{3}$ to identify the likely influence of stratospheric air. $\mathrm{CO}$ and $\mathrm{O}_{3}$ were detected by VUV-fluorescence and UV-absorption, respectively, with an accuracy of 5\% for both measurements. The instruments used have already been described (Gerbig et al., 1996; Schlager et al., 1997).

The Polar Nephelometer probe is designed to measure the optical parameters of clouds containing either water droplets or ice crystals or a mixture of these particles. The sensor measures the scattering phase function of an ensemble of cloud particles intersecting a collimated laser beam. For further details on the probe we refer to Gayet et al. (2002). In this study we used the data from this probe to identify periods where liquid water was present in the cloud.

The PMS FSSP-300 measures the number density of cirrus crystals from scattered light coming from ice crystals illuminated by laser light (Baumgardner et al., 1992). Because the ice crystals are not spherical a FSSP-300 size calibration for aspherical particles proposed by Borrmann et al. (2000) was used in this study. Application of this calibration in cirrus clouds results in 29 size classes measured by the FSSP-300 in the size range 0.38 to $15.8 \mu \mathrm{m}$. Due to noise the first three channels of the instrument showed not to be reliable during this experiment, and have therefore been excluded from the data interpretation. For this reason the minimum size class starts at $0.55 \mu \mathrm{m}$. At $1 \mathrm{~Hz}$ data resolution one count registered by the FSSP-300 corresponds to a crystal number density of about $0.2 \mathrm{~cm}^{-3}$.

Two complementary inlets were used to investigate the partitioning of aerosol particles in clouds: the aerosol inlet and the Counterflow Virtual Impactor (CVI). The aerosol inlet was used to sample the interstitial (non-activated particles left in between cloud elements) aerosol, or out-of-cloud (ambient) aerosol. The inlet simply consists of a $\frac{1}{4}$ inch stainless steel tube with the opening facing opposite the flight direction. Due to inertia, large particles $\left(D_{p}>1 \mu \mathrm{m}\right)$, pass by the probe and only non-activated particles are sampled (Schröder and Ström, 1997). The CVI separates inertially crystals larger than $5 \pm 1 \mu \mathrm{m}$ and smaller than about $60 \mu \mathrm{m}$ (aerodynamic diameter) from the surrounding atmosphere (Ogren et al., 1985). Once collected, ice crystals evaporate in the warm, dry and particle-free carrier air. The nonvolatile residues (at $25-30^{\circ} \mathrm{C}$ ) remaining from the crystals 
are characterized by sensors working downstream of the inlet. Due to the design of the CVI probe the sampled air is enriched compared to ambient conditions. During the two INCA campaigns the enrichment factor was typically abound 150, which was corrected for in the analysis. For the interpretation of the CVI data we assumed that each cloud particle leaves only one residual particle behind. Comparing the CVI to the PMS FSSP-300 probe has shown this assumption to be valid (Seifert et al., 2002).

Inside the aircraft, the sampling air coming from the aerosol and CVI probe is distributed between various instruments. In this study we focus on number density of the sampled particles, which was measured by two TSI 3010 condensation particle counters (CPCs). The total number densities of interstitial particles (Nint) and residuals (Ncvi) refer to the number densities of particles larger than 0.014 and $0.01 \mu \mathrm{m}$ in diameter, respectively. At $1 \mathrm{~Hz}$ data resolution one count registered by the CVI and the aerosol payload corresponds to a crystal number density of about $0.0004 \mathrm{~cm}^{-3}$ and $0.06 \mathrm{~cm}^{-3}$, respectively.

Data is stored at $1 \mathrm{~Hz}$, but when comparing different sensors, which have different response and lag times, this resolution is too high. To compensate for some of these problems and at the same time retain fine scale features, a $6 \mathrm{~s}$ moving average was applied to the data.

\subsection{Data selection}

The data set used in this study was selected to represent cirrus clouds in the upper troposphere and tropopause region only, and we limited our data to cirrus measurements above $6 \mathrm{~km}$ altitude and a temperature below $235 \mathrm{~K}$. According to theory this temperature range should be dominated by homogeneous nucleation (e.g. Pruppacher and Klett, 1997). We would like to point out that observations above $235 \mathrm{~K}$ contribute to less than $20 \%$ of the data in both hemispheres. The likely influence of stratospheric air was excluded when ozone mixing ratios were above $75 \mathrm{ppbv}$. To define in-cloud data we used the crystal number density given by the CVI since the dynamic range of the CVI is two order of magnitude larger than the range of the FSSP. In order to be classified as incloud data, the crystal number density measured by the CVI had to be above $0.001 \mathrm{~cm}^{-3}$. At this ambient number density the uncertainty due to counting statistics is $26 \%$. The mode or most typical crystal number density was around $1 \mathrm{~cm}^{-3}$ for both campaigns, which is well above the in-cloud criteria used in this study. In-cloud data points with the presence of liquid water were only observed down to $239 \mathrm{~K}$ by the Polar Nephelometer. After data reduction a total of approximately 24 and $10 \mathrm{~h}$ of observations remains from 32 and 17 flight hours performed in the Southern and Northern Hemispheres, respectively.

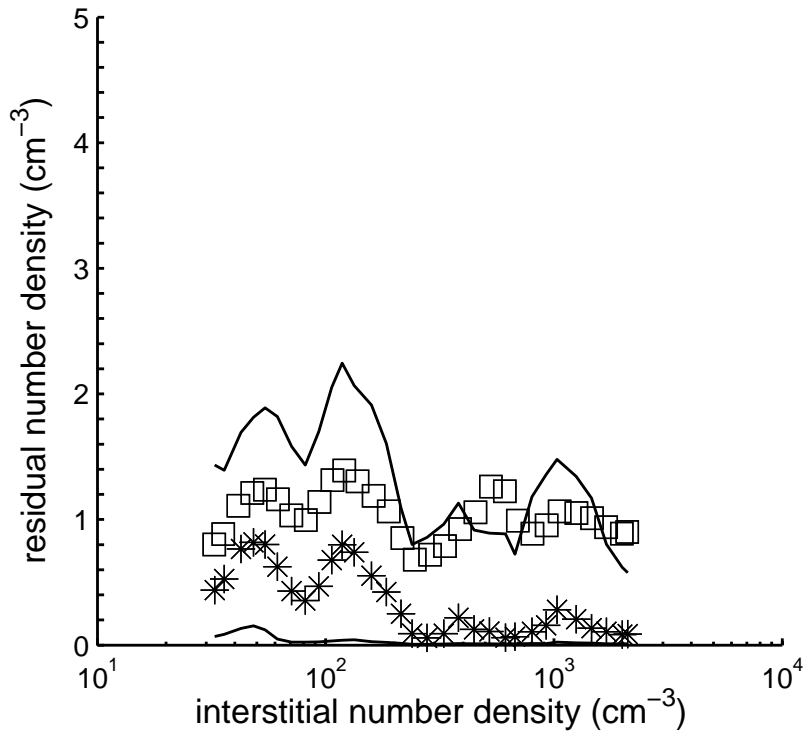

Fig. 1. Crystal residual number density as a function of interstitial aerosol number density. Averages are shown as squares and medians as stars. The two lines correspond to the 25th and 75th percentiles.

\section{Results}

Traditionally the sensitivity of warm clouds to changes in aerosol number density (Twomey effect) is studied by expressing the droplet number as a function of aerosol mass or cloud condensation nuclei $(\mathrm{CCN})$ number density. We proceed analogously with our data and plot the crystal number density as a function of aerosol number density. The data is classified according to Nint into bins. The bin limits are overlapping by $50 \%$, which generates a smoothing of the data. Average and median Ncvi are calculated for each bin. The result is presented in Fig. 1. The difference between averages and medians is relatively large, indicating a skewed distribution of the crystal number density data. The 25th and 75th percentiles document large variability in Ncvi. Apparently, there is no straightforward relation between aerosol and crystal number density in Fig. 1. Any signal of aerosol-cloud interactions sought for is expected to be small compared to the natural variability, but studying individual time series shows that more analysis is necessary before simply making a scatter plot between crystal number densities as a function of aerosol number densities. The response to changes in aerosol number densities may not always have the same sign, which will be illustrated below as an example for one flight segment.

3.1 Flight segment obtained during the 27 September 2000

The first mission flight of the INCA Prestwick campaign was dedicated to probe a cirrus field approaching from southwest. 


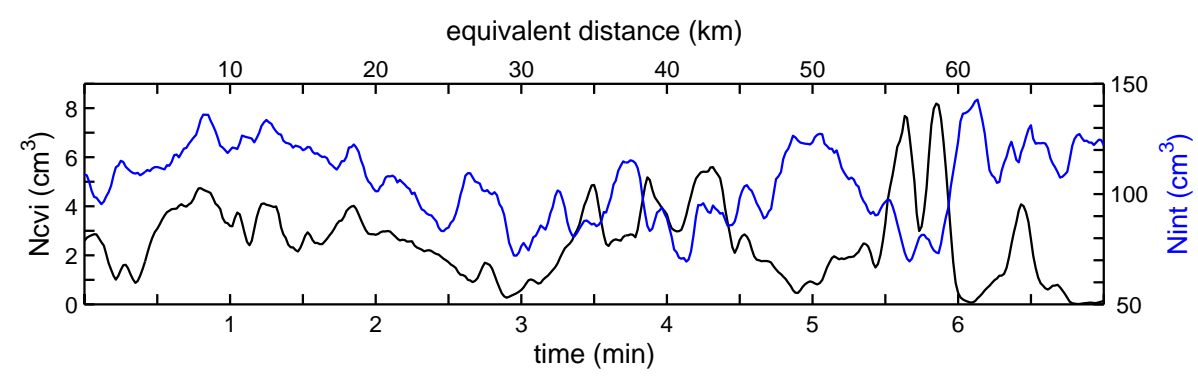

Fig. 2. Example of time series obtained during the INCA NH campaign on 27 September 2000. The starting point of the time series (time=0) corresponds to 13.94 UTC. Crystal residual number density (Ncvi, black line), interstitial aerosol number density (Ncvi, blue line). Data presented as $6 \mathrm{~s}$ moving averages.

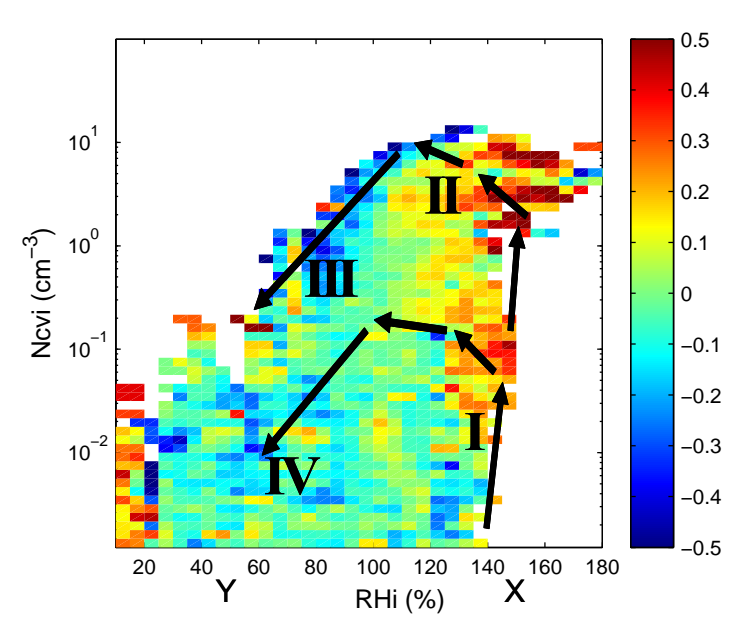

Fig. 3. Average vertical wind as a function of relative humidity over ice (RHi) and crystal residual number density (Ncvi). The color scale is in $\mathrm{ms}^{-1}$. The data corresponds to the NH. The Roman numbers indicate different phases in the cirrus lifecycle. $\mathrm{X}$ and $\mathrm{Y}$ are explained in the text.

After take-off the Falcon was heading north to $59^{\circ} \mathrm{N} 5^{\circ} \mathrm{W}$. From this turning point the cloud field top was entered at $10 \mathrm{~km}$ altitude. The aircraft continued approximately $30 \mathrm{~min}$ at this flight level. Figure 2 presents a short time series of the aerosol and crystal number densities obtained during this flight leg at constant altitude. The observed temperature was around $222 \mathrm{~K}$ and the relative humidity over ice varied between 90 and $140 \%$. For the first part of the cloud $(t<3 \mathrm{~min})$ Ncvi covariates with Nint. Local maxima in aerosol number density correspond to local maxima in crystal number density. From about 4 min and onwards Ncvi and Nint anticovariate. Clearly, local maxima in crystal number density correspond to local minima in aerosol number density and vice versa.

From Fig. 2 we learn that along a short flight segment, at constant altitude and temperature, and within the same air mass, we can observe both correlation and anti-correlation between Ncvi and Nint. Periods of correlation or anti- correlation in this flight segment appear on scales from a few seconds to a couple of minutes. Visual inspection of other flight segments from the two campaigns presented similar appearances. Locally over short flight segments the relation between aerosols and crystals can be very clear as in Fig. 2, but when all these segments are mixed together the signal is lost because positive and negative relations will cancel out on average. What determines when the aerosol-cirrus interaction is positive or negative in terms of changes in the particle number density?

We hypothesize that the aerosol-cloud relation observed in the time series is related to what particular phase of the cloud lifecycle the measurements are performed at. Whether the cloud is just forming, has reached a mature state, or is in the final phase just about to dissolve completely, could be of importance to the observed aerosol-cloud relation.

\subsection{Correlation analysis}

Ström et al. (2002) suggested a novel way of presenting data that relates an observed parameter to the cloud lifecycle. The investigated parameter is simply plotted as a function of relative humidity and crystal number density. The idea behind this way of plotting is illustrated using the vertical wind data presented in Fig. 3. Observations of vertical wind are binned according to crystal number density in logarithmically equidistant bin limits, and according to RHi in steps of $5 \%$ with a bin width of $10 \%$ units (0-10, 5-15, etc). This is done to provide some smoothing to the data. In each of these two-dimensional bins at least five data points must be present or else the bin is given the code "no data". The average vertical wind in each bin is color coded, such that warmer colors represent stronger updrafts, and colder colors represent stronger downdrafts. At large, warm colors are predominantly found above and cold colors below ice saturation. There is also a gradient towards warmer colors above as well as colder colors below ice saturation as the crystal number density increases. Around ice saturation the colors are mostly in the green indicating no preference for either updraft or downdraft. Data at the very lowest relative humidities should be considered with caution since it may arrive 
from flight segments across cloud edges and where instrument memory effects may cause artifacts. Using the data in Fig. 3 we can walk through the lifecycle of cirrus clouds.

Cirrus clouds form in ice supersaturated environments and dissolve when the relative humidity decreases below ice saturation. Just prior to the formation of the cloud, relative humidity is high and there are no crystals present. This could for instance be $X$ in Fig. 3. Ice particles form, yet in the beginning of the cloud they are limited in number and size, and do not affect the ambient relative humidity to any extent. In fact relative humidity may still be increasing even after the first crystals appear. This would be region I in Fig. 3. As ice mass is produced in the cloud the crystals begin to consume available water vapor, which reduces the relative humidity and stops further nucleation of ice crystals. The crystals continue to grow until ice saturation is reached, which is illustrated by the arrows in the figure. The more vigorous the cloud, the higher the relative humidity and the higher the crystal number density will be. This would be region II in Fig. 3. Once the humidity drops below ice saturation the ice crystals start to evaporate, which is marked III and IV in Fig. 3. Eventually, all the crystals will have evaporated completely and the cloud has disappeared. This would be $\mathrm{Y}$ in the figure.

Our aim is to plot the correlation coefficient between Nint and Ncvi analogous to the vertical wind in order to find out if there are regions where aerosols and ice crystals are positively or negatively correlated. First we need to determine a time window to calculate the correlation over. Small time windows correspond to small horizontal extents and the data within the time window to a greater extent share external parameters such as temperature, humidity, and dynamic forcing. As the time window increases the conditions become less homogenous and the correlation is calculated over different phases of the cloud lifecycle and even different air masses. Based on time series such as the one presented in Fig. 2, correlation and anti-correlation between Nint and Ncvi lasts from seconds up to several minutes, and using time windows much longer than a few minutes is not useful. Longer time windows reduce the amount of data, as this will generate fewer continuous in-cloud flight segments. Starting from a $10 \mathrm{~s}$ window we subsequently increased the time window up to $390 \mathrm{~s}$. This time window is then allowed to move over the data to generate a new parameter, which is a moving correlation coefficient . To keep as much data as possible we allow for a $5 \mathrm{~s}$ gap in the time series. The average correlation coefficient is plotted as a function of the time window in Fig. 4. From insights studying Fig. 3, illustrating the strong separation in updraft and downdraft, we split the data into above and below ice saturation. The average correlation coefficients are very small, but the ice supersaturated data show higher values and a local maximum at about $100 \mathrm{~s}$. Although the absolute values of the average correlation coefficient are small we accept a time window of $100 \mathrm{~s}$ as the best for our purpose. At typical airspeeds of the Falcon aircraft this time

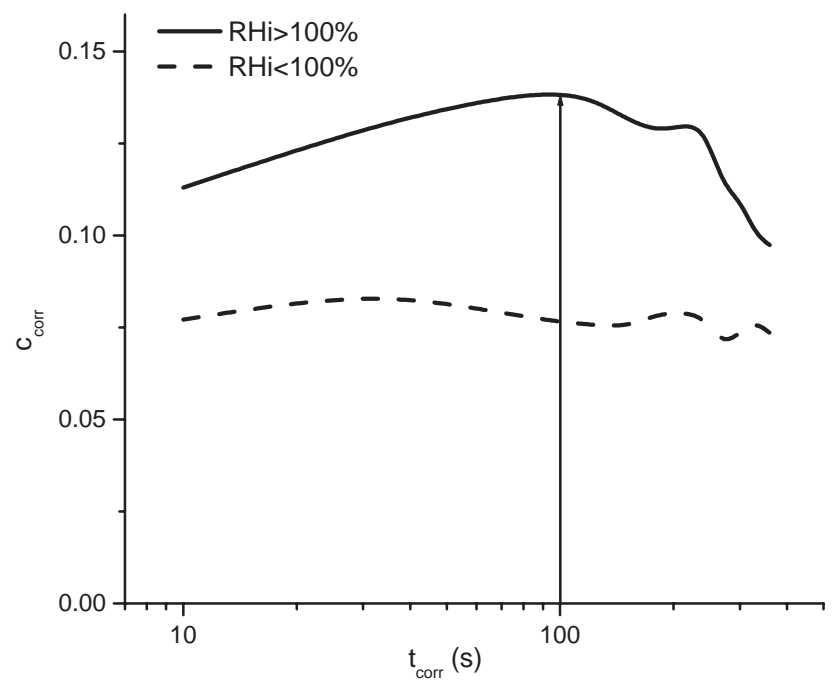

Fig. 4. Average moving correlation coefficient between interstitial aerosol and crystal residual number density as a function of the time window over which the correlation is calculated (solid line, $\mathrm{RHi}>100 \%$ data; dashed line, $\mathrm{RHi}<100 \%$ data).

window corresponds to a horizontal distance of ca. $20 \mathrm{~km}$, which is in the same range as the mesoscale cirrus generating cells discussed by Sassen et al. (1989).

We know from studies by Minikin et al. (2003) and Baehr et al. (2003) that the ambient air is significantly different in the Northern and Southern midlatitudes considering aerosols and trace gases. For this reason, it is of interest to compare cirrus observations from the two campaigns in order to investigate if there are any differences or similarities. In Fig. 5 the average correlation is presented as a function of Ncvi and $\mathrm{RHi}$, divided into the two campaigns.

The colors are mostly in the green, which indicates coefficients between -0.2 and 0.2 , but there are also regions with colder or warmer colors, especially for the SH-data. Along the forming branch (regions I and II in Fig. 3), frequently presents values above and some times much above 0.5 . The real significance is not the magnitude itself but the fact that the colors appear in regions. If did not relate to relative humidity and crystal number density, we would expect a non-coherent mosaic pattern of colors. The colors are not as strong for the NH-data. There is a weak streak of red and yellow in the forming branch. However, the evaporating regions (III and IV in Fig. 3) are tinted more to the blue indicating more pronounced negative correlations. Figure 5 shows that cloud formation is more prone to positive correlations whereas the correlations are smaller and even negative at places in the dissolving cloud. The correlation analysis above does not show where aerosol number densities are high or low, only where ice crystals and aerosol number densities correlate. In the next section we continue to make use of the plotting format introduced in Fig. 3, and study the interstitial aerosol number density. 

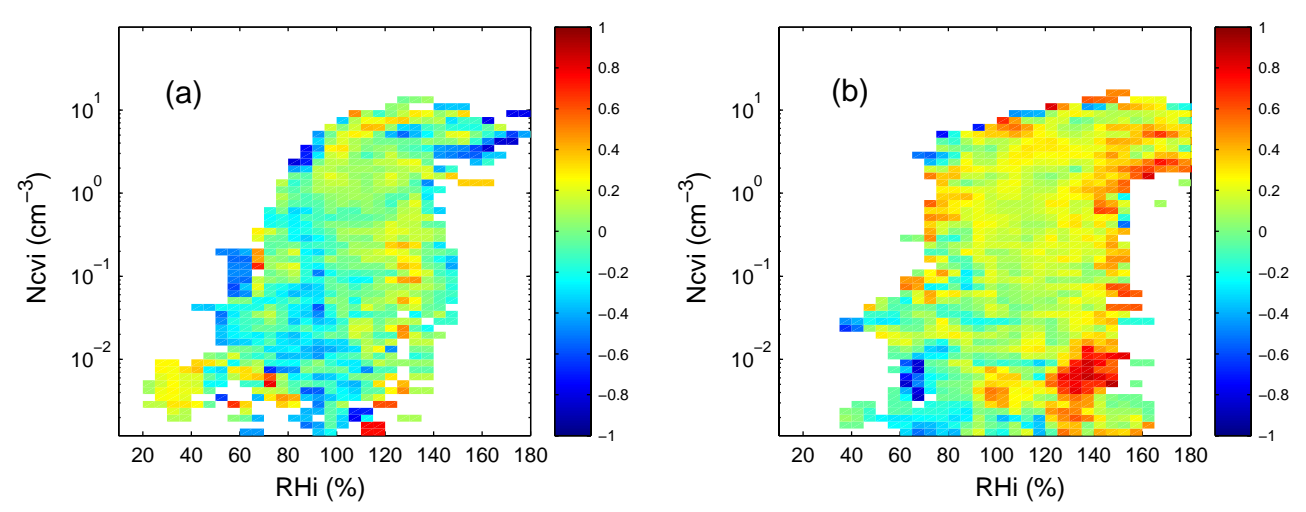

Fig. 5. Average moving correlation coefficient between interstitial aerosol and crystal number density as a function of crystal residual number density (Ncvi) and relative humidity over ice (RHi). Correlation coefficients have been derived for a $100 \mathrm{~s}$ time window. (a) NH-data, (b) SH-data.
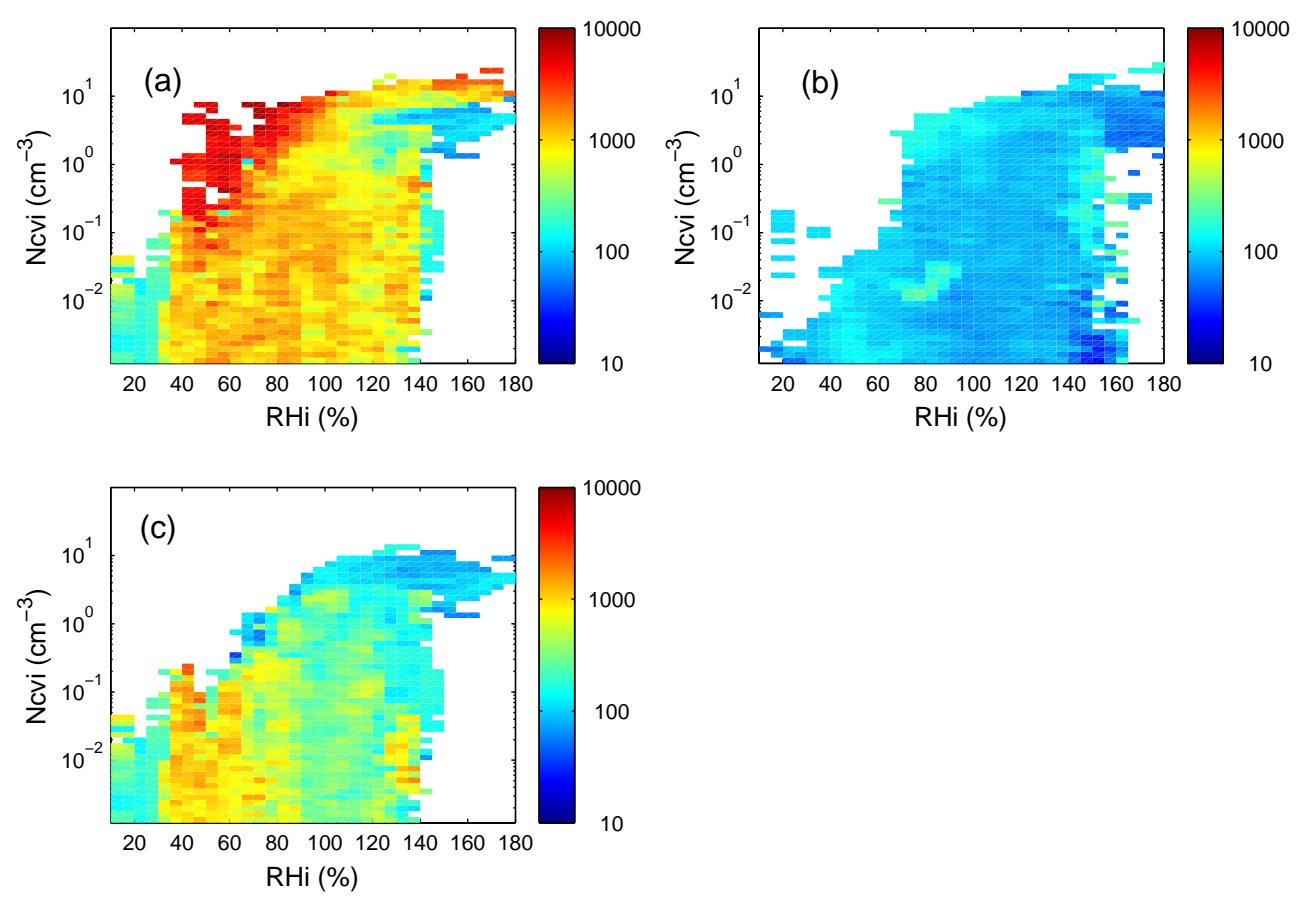

Fig. 6. Average interstitial aerosol number density as a function of crystal number density (Ncvi) and relative humidity over ice (RHi). The color scale is in $\mathrm{cm}^{-3}$. (a) $\mathrm{NH}$-data, (b) $\mathrm{SH}$-data, (c) $\mathrm{NH}$-data, $\mathrm{CO}<90 \mathrm{ppbv}$.

\subsection{Interstitial aerosol number density}

Figures $6 \mathrm{a}$ and $\mathrm{b}$ show the average interstitial aerosol number density as a function of relative humidity and crystal number density. In a world where aerosols do not affect clouds, nor are affected by clouds, we would expect a uniform color over the whole domain. This appears to be the case for the SH-data and also the NH-data almost gives this appearance except for the region of very high Nint in the upper left part of the plot and a region of low Nint at high RHi. As expected the colors for the NH-data are warmer due to the higher pollution level in the NH (Minikin et al., 2003).
We know from meteorological data and notes taken by the operator during the flights, that some of the high aerosol number densities were measured in the vicinity of anvils originating from the outflow of convective clouds. Deep convection can loft boundary-layer air, which is rich in aerosol particles and precursors gases associated with polluted air near the surface, into the upper troposphere. Although this may be an important mechanism for altering the atmospheric composition in the tropopause region it is not the main focus of this study and therefore we exclude these observations from the analysis. 

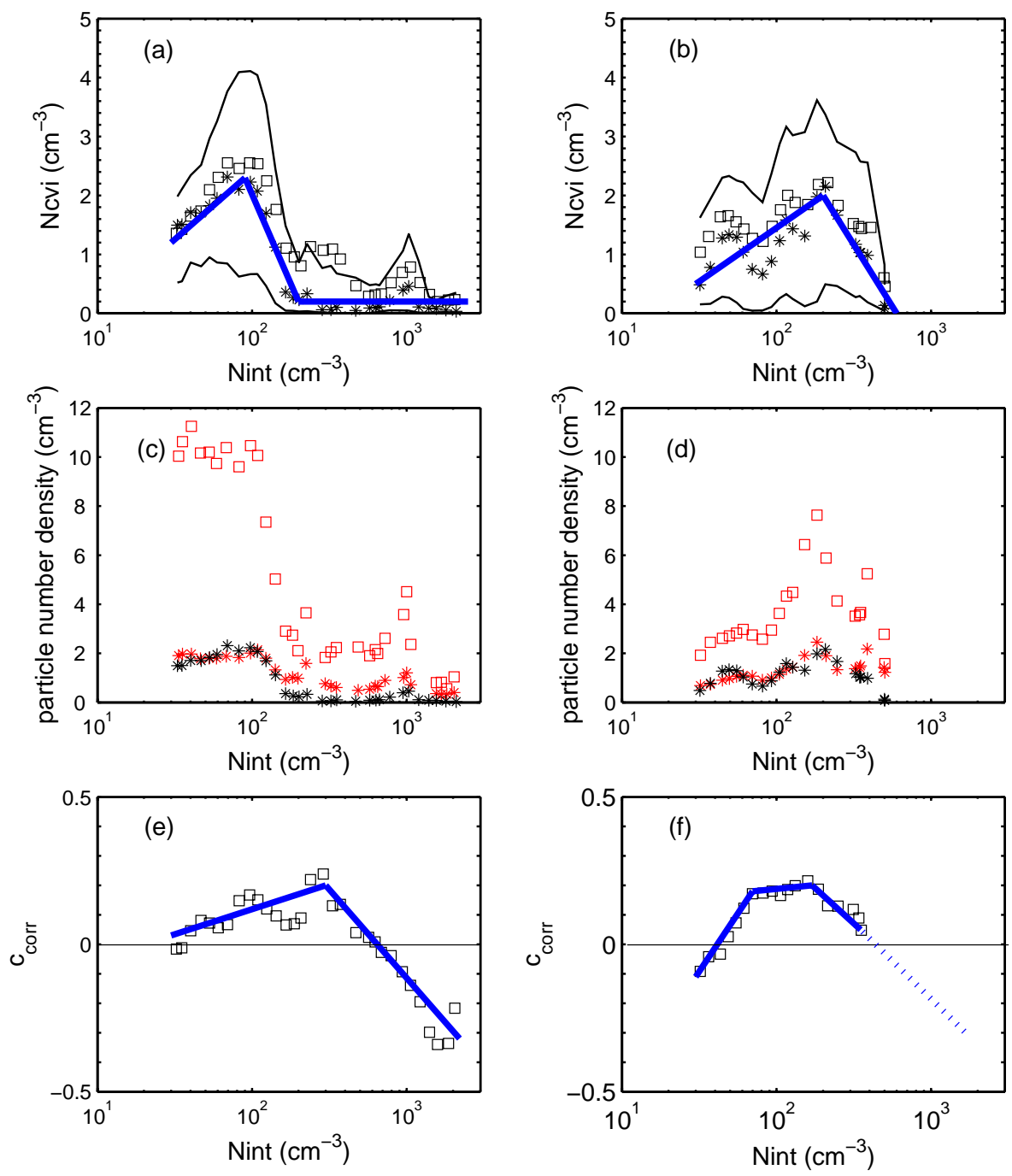

Fig. 7. Upper two panels: Crystal residual number density (Ncvi) as a function of interstitial aerosol number density (Nint). Averages are shown as squares and medians as stars $(\mathrm{RHi}>100 \%, \mathrm{CO}<90 \mathrm{ppbv})$. The two thin lines correspond to the 25th and 75 th percentiles. (a) Data from NH, (b) Data from SH. Middle two panels: Median particle number density given by CVI (Ncvi, black stars), the integral of the FSSP-300 size distribution measured in the size range 1-4 $\mu \mathrm{m}$ (NFSSP-1, red squares) and the integral of the FSSP-300 size distribution measured in the size range 4-15.8 $\mu \mathrm{m}$ (NFSSP-2, red stars) (RHi $>100 \%, \mathrm{CO}<90$ ppbv). (c) Data from NH, (d) Data from SH. Lower two panels: Average moving correlation coefficient between interstitial aerosol and crystal residual number density as a function of interstitial aerosol number density (Nint). Correlation coefficients have been derived for a $100 \mathrm{~s}$ time window (RHi $>100 \%, \mathrm{CO}<90 \mathrm{ppbv})$. (e) Data from NH, (f) Data from SH. Blue lines correspond to our interpretation of the slopes. The dashed blue line is simply an extrapolation.

Baehr et al. (2003) have shown for the INCA data that the mean CO mixing ratio is decreasing with altitude over Prestwick. Hence a high CO mixing ratio at high altitudes is an indicator of recent transport of polluted boundary layer air. As a threshold level we used the highest $\mathrm{CO}$ value observed in the SH campaign, which was 90 ppbv. Both data sets may of course still be influenced by convective transport, but at least the influence from recent transport of polluted air is limited.

After the high CO data has been removed the new NH-data presented in Fig. $6 \mathrm{c}$ shows some interesting features. First of all, the data to the upper left of Fig. 6a is now gone and we learn that the observations of high crystal number densities at low relative humidities are linked to recent transport of polluted boundary layer air. Secondly, the color pattern now presents a clear gradient from low Nint at high RHi and high Ncvi to high Nint at low RHi and low Ncvi. This trend could be indicative of a cloud effect on the aerosol (where crystals remove aerosols through scavenging), or, alternatively, of a source of aerosol particles as the cloud evaporates. We will return to this issue in Sect. 3.5. 


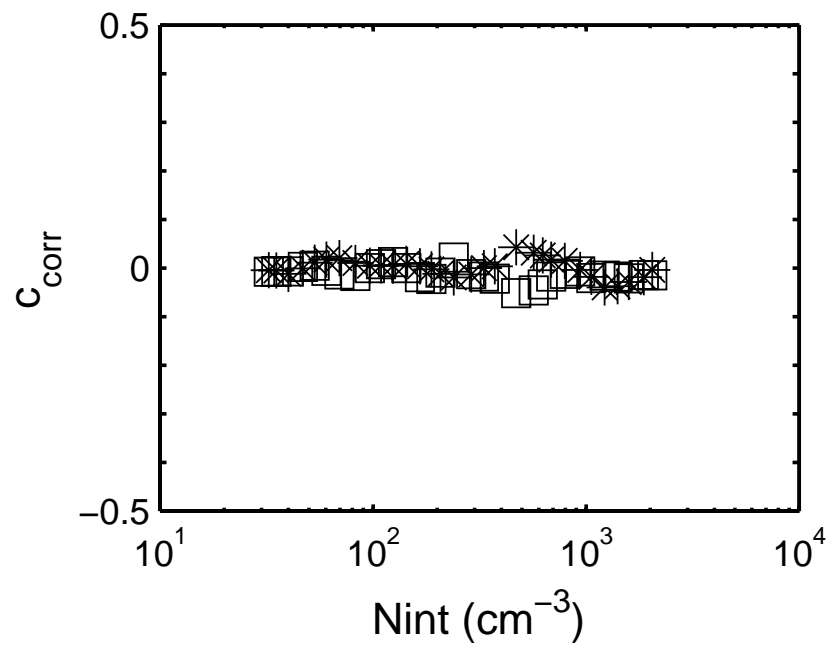

Fig. 8. Average moving correlation coefficient between interstitial aerosol and crystal residual number density as a function of interstitial number density (Nint). Correlation coefficients have been derived for a $100 \mathrm{~s}$ time window ( $\mathrm{RHi}>100 \%, \mathrm{CO}<90 \mathrm{ppbv})$. $\mathrm{NH}$ Nint data and simulated Ncvi data. a) Ncvi was randomly chosen in the interval 0.001 to $10 \mathrm{~cm}^{-3}$ (squares), (b) Ncvi was randomly distributed but was weighted to the observed NH Ncvi distribution (stars).

\subsection{Ice crystals vs. aerosols}

We again plot the crystal number density as a function of aerosol number density as in Fig. 1, but based on the insights gained from the analysis conducted above we also limit the data to humidities above $100 \%$ RHi and exclude situations where the $\mathrm{CO}$ concentration exceeds 90 ppbv. We also split the data into the two different campaigns ( $\mathrm{NH}$ and $\mathrm{SH}$ ). The resulting relations are plotted in Figs. 7a and b. Clearly, averages and medians are much closer together compared to Fig. 1, which shows that the distribution of data points for a given Nint is less skewed than previously. The quartiles represented by the two thin lines are still far apart showing that there is still substantial variability in the data. The blue lines represent our interpretation of the data. The data sets $(\mathrm{NH}$ and $\mathrm{SH}$ ) are similar in that they both show local maxima in the Nint range 100 to $200 \mathrm{~cm}^{-3}$, where the SH-data is shifted towards the higher value. The slopes to the left in the figures correspond to a 3 to 4 times increase in the crystal number density in relation to a tenfold increase in the aerosol number density. As Nint increases beyond the ca. 100 to $200 \mathrm{~cm}^{-3}$ the mean crystal number density decreases at about the same rate for both data sets. For much higher aerosol number densities, only present in the NH data set, the mean Ncvi remains low.

When interpreting crystal residual data we have to keep in mind that the CVI only detects particles larger than ca. $5 \mathrm{~m}$ in size, and even if the cloud is composed of a large number of crystals these will not be detected should the size be too small. Thus, there is a possibility that the crystal number density continues to increase with increasing Nint, but remains undetected by the CVI as the mean size is smaller than the detection limit of the CVI. To address this issue and to verify the in Figs. 7a and b found relations between Ncvi and Nint we extend our analysis by including FSSP-300 crystal size distribution data. A lower limit of $4 \mu \mathrm{m}$ for the FSSP size distribution has shown to give the best agreement between FSSP and CVI data. Integrating the FSSP size distribution from 4 to $15.8 \mu \mathrm{m}$ (NFSSP-2) allows us to emulate a CVI with respect to the cut-off size. Recall that the CVI selects particles based on the aerodynamic properties of the crystal whereas the FSSP-300 inverts the light from a particle based on assumed optical properties of the crystal. To get a measure for the number density of small ice particles we integrate the FSSP size distribution from 1 to $4 \mu \mathrm{m}$ (NFSSP-1). The lower integration limit was chosen to be above the lower detection limit of the FSSP to reduce the effect that large aerosol particles might have on the FSSP-300 data. In Figs. 7c and $d$ we plot NFSSP-1, NFSSP-2 together with Ncvi as a function of interstitial aerosol number density. When interpreting FSSPdata we have to keep in mind the FSSP data is biased towards low crystal number densities due to counting statistics. Nevertheless close agreement between NFSSP-2 and Ncvi data is found over the entire Nint range highlighting the consistency of FSSP and CVI measurements yet based on totally different physical principles. The NFSSP-1 data is shifted towards high number densities but resembles the same main features as found for NFSSP-2 and Ncvi; high crystal number density at low Nint and low crystal number density at high Nint. Hence the number of small ice crystals particles does not appear to increase with increasing aerosol number density, but rather mimics the trend for the larger crystals.

Data in Figs. 7a-d shows how the mean crystal number density changes as a function of Nint, but we do not know how the sensitiveness is distributed over the data. In other words, one could imagine that the average crystal number density is high for a given Nint, but the sensitiveness to changes in the aerosol number density may be low. That is, the correlation between Nint and Ncvi would be weak at a given Nint. We use the moving correlation coefficient between Ncvi and Nint derived previously and plot it as a function of Nint, which is presented in Figs. 7e and $\mathrm{f}$. As in Figs. 7a and $b$ the blue lines represent our interpretation of the slopes. Average increases with increasing Nint in the $\mathrm{NH}$-data up to ca. $300 \mathrm{~cm}^{-3}$. Above this, decreases and at around $600 \mathrm{~cm}^{-3}$ correlations become negative. Although the SH-data starts out with negative values of at low Nint the appearance with a local maximum is similar. If the slope to the right is extrapolated (dashed line), the intercept of the zero line (ca. $500 \mathrm{~cm}^{-3}$ ) is similar to the $\mathrm{NH}$-data.

We test if it is possible to achieve an apparent relation between the correlation and the aerosol number density simply by chance. We take our measured Nint time series and assign to each data point a random crystal number density. We 

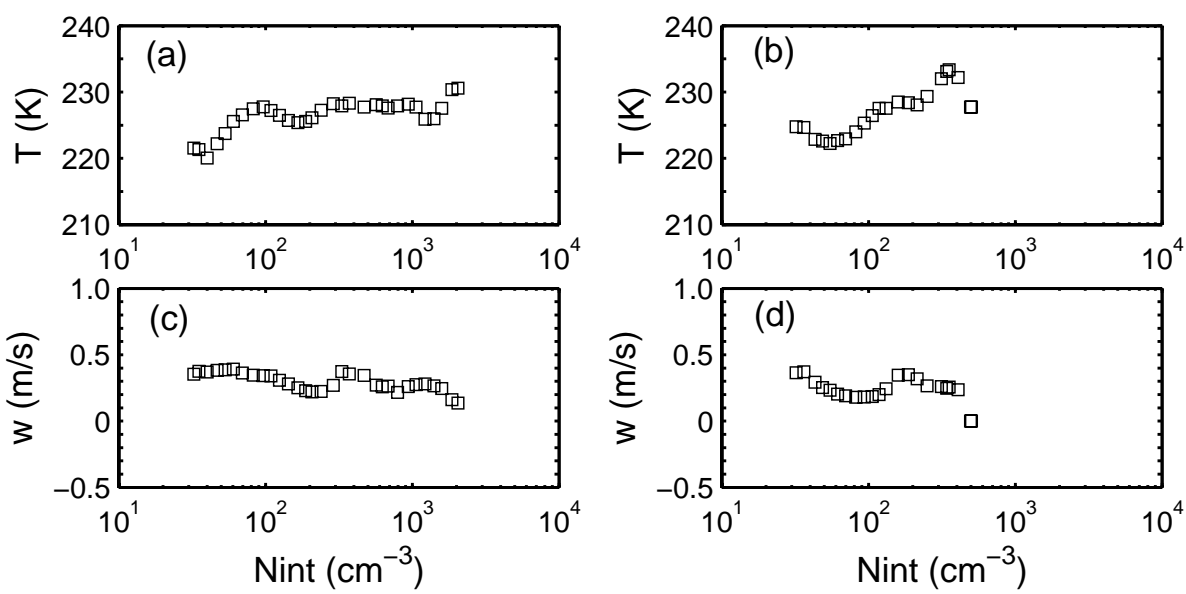

Fig. 9. Average temperature $(\mathrm{T})$ and average vertical wind $(\mathrm{w})$ as a function of interstitial aerosol number density (Nint). Data from the NH $(\mathbf{a}, \mathbf{c})$, and from $\mathrm{SH}(\mathbf{b}, \mathbf{d})$. (RHi $>100 \%, \mathrm{CO}<90 \mathrm{ppbv})$.

then calculate a correlation coefficient as was done for the original data. Two simulations were performed. In the first simulation, a crystal number density was randomly chosen in the interval 0.001 to $10 \mathrm{~cm}^{-3}$. In the second simulation, the crystal number density again was randomly distributed but this time it was weighted to the total observed crystal number density distribution. In both cases the average correlation became just about zero for all Nint bins as shown in Fig. 8 for the NH-data.

We know from numerical simulations that cloud microphysical properties are controlled by environmental conditions such as temperature and vertical wind. Kärcher and Lohmann (2002) showed that changing the vertical wind form 0.1 to $1 \mathrm{~m} / \mathrm{s}$ can increase the simulated ice crystal number density by two orders of magnitude. Increasing temperature on the other hand increases the ice particle growth rate and the depletion of excess water, hence freezing is terminated earlier resulting in lower crystal number densities. In our case we have tried to isolate the role of aerosols keeping everything else constant. The last statement is of course very important considering the sensitivity of microphysical properties to changes in temperature and vertical wind. As long as the distributions of vertical wind and temperatures are the same over different Nint, the crystal-aerosol relation should not be influenced by these environmental conditions. For completeness we have plotted the average vertical wind and average temperature as a function of Nint in Figs. 9a-d. From the figures one can see that the average values are not constant throughout the Nint range and there are likely differences in the distributions for any two given Nint as well. Subtle changes in vertical wind distributions may be very important for the mean crystal number density (Kärcher and Ström, 2003). Nevertheless, there are no obvious trends that would explain the observed relations with local maxima in Ncvi and the correlation coefficient in both data sets as presented in Fig. 7. The mean temperature increases with increasing Nint, which is explained by the fact that both variables decrease with altitude (recall that we removed data points where $\mathrm{CO}$ exceeded $90 \mathrm{ppbv}$ ). The mean vertical wind is never negative, which can be understood from Fig. 3 and from the fact that only data above $100 \%$ RHi was used.

\subsection{Cloud evaporation}

In Sect. 3.3 we observed a trend towards higher average Nint as the relative humidity decreased below ice saturation. On average the aerosol number density increases from 80 to $100 \mathrm{~cm}^{-3}$ and from 180 to $470 \mathrm{~cm}^{-3}$ as the humidity decreases from $120 \%$ to $80 \%$ for the $\mathrm{SH}$ and $\mathrm{NH}$, respectively. Several processes may affect the aerosol number density in a cloud: ice nucleation, precipitation and scavenging of aerosol particles. To begin with, aerosol particles are incorporated in ice crystals during cloud formation. However, since typically less than $1 \%$ of the ambient aerosol particles become scavenged in cirrus crystals as part of the formation process (Seifert et al., 2002) and only few ice crystals will grow large enough to precipitate, nucleation scavenging is not likely to significantly change the number of aerosol particles. Cirrus crystals may scavenge aerosol particles from the interstitial air due to Brownian diffusion. The effect of this potential process can be estimated by assuming a crystal diameter of $32 \mathrm{~m}$ and aerosol sizes as observed for the average interstitial distribution in the $\mathrm{NH}$ using a simple coagulation model (Ström et al., 1992). The number density of the crystals and the interstitial particles is taken to be 3 and $400 \mathrm{~cm}^{-3}$, respectively. Given these conditions, a cloud presence of $3 \mathrm{~h}$ changes the interstitial number density by around $10 \%$ for particles larger than $10 \mathrm{~nm}$ in diameter. Note that this approximation assumes no other processes such as mixing. For the SH-data this process is even slower due to the significantly lower level of aerosol loading. Hence we expect only small or negligible effects on the aerosol number density from scavenging by crystals. 

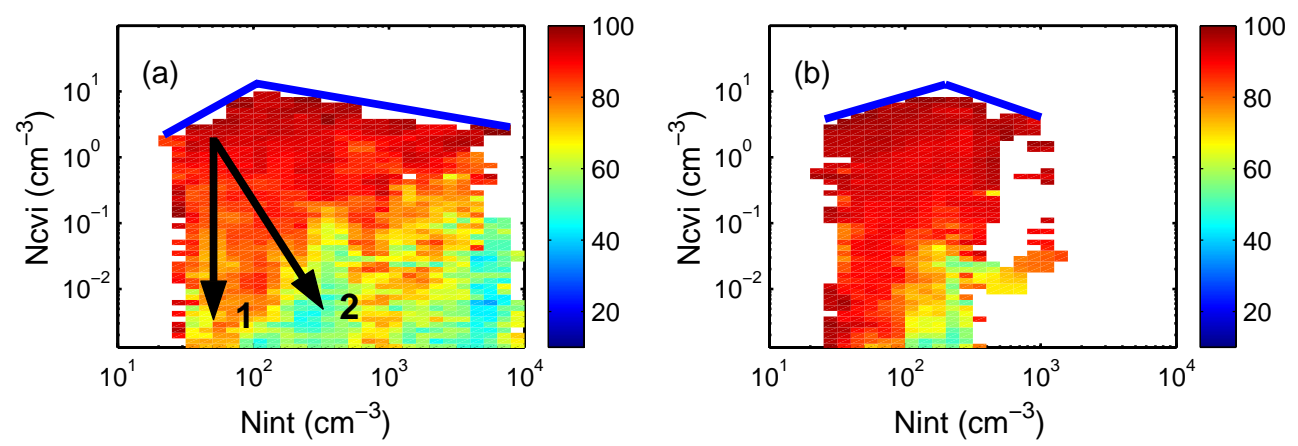

Fig. 10. Relative humidity over ice as a function of interstitial aerosol number density (Nint) and crystal residual number density (Ncvi) for evaporating cirrus $(\mathrm{RHi}<100 \%, \mathrm{CO}<90 \mathrm{ppbv})$. The color scale is in percent. (a) NH-data, (b) SH-data. The two black arrows indicate two different plausible pathways during evaporation. The two blue lines are tangent to the maximum crystal residual number density as a function of Nint.

From having focused on conditions where RHi is above $100 \%$ we now turn to the sub-saturated conditions. In Fig. 10 the average relative humidity less than $100 \%$ RHi is plotted as a function of Ncvi and Nint. As previously, data where $\mathrm{CO}$ exceeds $90 \mathrm{ppbv}$ is removed. The highest relative humidities (close to 100\%) are found at the highest crystal number density for any given Nint. This makes sense since the cloud has just started to evaporate and the crystal size is affected prior to a reduction in the number density. As the air becomes increasingly dry, the crystal number density decreases.

There are two alternative ways of interpreting Fig. 10. First, we can chose to keep the Nint constant and move from high to low Ncvi (arrow marked \#1 in the figure). The implication of this is that at low Nint values the cloud disappears at yet high relative humidities (warm colors). In other words, evaporation is quick (the cloud has disappeared if the crystal number density is less than $0.001 \mathrm{~cm}^{-3}$ ). At high Nint values the cloud does not disappear until the humidity becomes significantly lower (colder colors). This implies that clouds in an environment containing high aerosol number density evaporate more slowly than clouds in an environment where the aerosol number density is low. Secondly, we may move along the humidity gradient, which would make the evaporation rates more similar (arrow marked \#2 in the figure). However, this would imply that the aerosol number density would increase as the cloud evaporates. Although, Nint does not extend to particularly high values in the $\mathrm{SH}$, both data sets show the same tendency with respect to the color coding in Fig. 10. Both interpretations would explain the higher mean Nint at sub-saturated conditions as observed in Fig. 6. However, our data set does not lend us to determine which of these might be correct. Another interesting feature of Fig. 10 is that the maximum Ncvi as a function of Nint shows a similar local maximum as mean Ncvi did in Fig. 7. This is indicated by blue lines in Fig. 10.

\section{Discussion}

The observations presented above show a response of the correlation between the number density of crystals and aerosol particles to the developing stage of a cloud. Cloud formation was found to be more prone to positive correlations whereas smaller and even negative correlations were evident in dissolving clouds.

For cirrus clouds in the forming stage of their lifecycle the observed weak positive relation may be equivalent to the socalled Twomey effect for warm clouds. Unlike warm clouds, where the response of cloud elements to changes in aerosol number density is relatively well understood, we observed a rather complex relationship when expressing the crystal number density as a function of the aerosol number density. For aerosol number densities up to one or two hundred per cubic centimeter the number density of crystals was found to increase with the number density of interstitial particles. Above this level further increase in interstitial number density resulted in a decrease in the crystal number density. This was consistent in both campaigns.

Why is there an initial positive relation between Nint and Ncvi which then turns into a negative one? Since the role of aerosols in the formation of clouds is still an open issue we are left to speculate what impact changes of aerosol number density have on atmospheric ice particle number densities. It might be that the number of interstitial aerosol particles is a proxy for the number of particles suitable for ice crystal formation. At low Nint ice crystal formation might be limited by the number of suitable aerosol particles, causing a positive relation between Nint and Ncvi. However, this trend cannot last indefinitely as the water vapor supply in the upper troposphere is limited, which is why cirrus formation tends to be self-limiting. The higher the number of suitable ice nucleating aerosols, the more particles will compete for the available water causing a decrease of the peak saturation. This will in turn lead to a decrease of the crystal number density, since fewer particles will simply grow to become real 
ice crystals and will remain as haze drops or ice embryos. At some point (or Nint) we therefore reach a situation where no more ice crystals can be formed, marking the maximum crystal number density for a given dynamical forcing. A further increase in Nint will quench ice nucleation increasingly, resulting in a negative trend between Nint and Ncvi. Note that a negative relation between crystal number density and interstitial aerosol number density at high Nint was observed for both CVI and FSSP data. Hence the possibility that the crystal number density may continue to increase with increasing Nint, but remains undetected by the CVI as the mean size is smaller than the detection limit of the CVI, can be dismissed.

Are our findings consistent with theory? Numerical models simulating homogenous ice nucleation show that crystal number densities depend only weakly on aerosol number densities (e.g. Jensen and Toon, 1994; DeMott et al., 1994; Kärcher and Lohmann, 2002). Kärcher and Lohmann (2002) showed relative changes in crystal number density induced by realistic changes in the aerosol size distribution parameters to be small $(<30 \%)$ in most cases, except for the highest updraft velocities. The observed trends presented in this study are not very strong either. A tenfold increase in aerosol number density corresponds to a factor 3 to 4 increase in crystal number density. One of the interesting aspects of this study is that the mean crystal number density decreases as the aerosol number density increases beyond a few hundred per cubic centimeter. The correlation coefficient at high Nint in the $\mathrm{NH}$ even turns negative. Negative correlation between Nint and Ncvi was also observed in the study by Kärcher and Lohmann (2002), however only in simulations where the width of the aerosol size distribution was increased. If other modes of nucleation such as heterogeneous freezing are active, the response to changes in aerosol size distribution may be different. Jensen and Toon (1997) concluded that heterogeneous freezing on relatively few insoluble particles $\left(\mathrm{N}<0.1 \mathrm{~cm}^{-3}\right)$ should result in fewer ice crystals than if homogeneous nucleation was to occur, whereas if large numbers of insoluble particles are abundant the crystal number density may be increased. More recently Kärcher and Lohmann (2003) have emphasized that a pronounced indirect aerosol effect on cirrus is possible when adding efficient ice nuclei to liquid sulfate aerosols. Nevertheless very little is known about the Twomey effect of ice clouds when allowing for heterogeneous freezing.

When discussing the Twomey relation for cirrus clouds we have to keep in mind that the indirect aerosol effect depends not only on the anthropogenic aerosol, but also on the preindustrial aerosol burden. Anthropogenic aerosols such as sulfate or carbonaceous aerosols have substantially increased the global burden of aerosols from pre-industrial days to the present day. However, since there are no observations of preindustrial clouds and aerosols available, we do not know to what extent even the pristine environments, such as the region around Punta Arenas, have been effected.
When it comes to cirrus under evaporation, we found the interstitial number density to increase with decreasing crystal number density and relative humidity. Plotting the average relative humidity as a function of interstitial and crystal number density for evaporating cirrus $(\mathrm{RHi}<100 \%)$, presents two alternative interpretations: (1) air pollution (high aerosol number density) retards cloud evaporation, and (2) cloud evaporation is associated with a source of aerosol particles. We do not know which of the two interpretations, if any, is correct, thus leaving room for further speculation.

Assuming that the amount of aerosol particles is a proxy for the degree of pollution, cirrus crystals may incorporate solutes onto the crystal which are produced by gas phase reactions or heterogeneously on the particle surface. When a crystal is relatively large this surface contamination may have little influence on the transfer of water vapor to or from the crystal as the relative humidity changes. As the crystal evaporates and becomes smaller the presence of this surface contamination, or impurity, may become important. The threshold could be reached at a level where one or a few monolayers are covering the evaporating crystal preventing water vapor to have full access to the ambient environment. In other words, surface effects may suppress evaporation rates which would be consisted with results by Chen and Crutzen (1994). Chen and Crutzen (1994) studied the possible influence of trace chemicals on the growth and lifetime of ice crystals, based on the concept of a transition layer on the crystal surface. They showed theoretically that ice crystals may survive without complete evaporation at relative humidities over ice as low as $91 \%$.

The other interpretation refers to the possible production of particles. As pointed out above, ice crystals may scavenge chemical species from the interstitial air. When an ice crystal evaporates, the release of the scavenged species may result in locally enhanced concentrations which could favor aerosol formation. The evaporating cloud, as a source of particles, could explain the shift towards more negative correlations below ice saturation in Fig. 5. If this particle production mechanism is real, cirrus clouds could constitute a very significant source of new particles in the tropopause region. Note that in the CVI crystals are transported in nitrogen gas covered from sunlight. Hence we don't expect particle production to be possible within the CVI from the same mechanism.

\section{Summary and conclusions}

The intention of this study was to investigate the sensitivity of crystal number density to changes in aerosol number density. Following conclusions can be drawn from the results:

1. Aerosol cirrus interactions can be linked to different phases of a cirrus lifecycle. Cloud formation is largely associated with positive correlations between the number density of aerosols and crystals whereas the 
correlations are smaller or even negative in a dissolving cloud.

2. For cirrus clouds under formation the observed weak positive relation may be equivalent to the so-called Twomey effect for warm clouds. Expressing the crystal number as a function of the aerosol number density shows a rather complex relationship. The data sets are similar in that they both show local maxima in the Nint range 100 to $200 \mathrm{~cm}^{-3}$, where the SH-data is shifted towards the higher value. For lower number densities Nint and Ncvi are positively related. The slopes in the data suggest that a tenfold increase in the aerosol number density corresponds to a 3 to 4 times increase in the crystal number density. As Nint increases beyond the ca. 100 to $200 \mathrm{~cm}^{-3}$, the mean crystal number density decreases at approximately the same rate in both data sets. At much higher aerosol number densities, only present in the NH data set, the mean Ncvi remains low.

3. There seems to exist a relationship between aerosol and ice crystals even in evaporating cirrus. Our data shows the number density of aerosol particles to increase with decreasing relative humidity and crystal number density. We offer the following two possible interpretations:

- Evaporating clouds are associated with a source of aerosol particles.

- Air pollution (high aerosol number density) retards evaporation rates.

We note, however, that both interpretations must be regarded as speculative at this point. In this study we did not address the role of the chemical composition of the particles. Differences in the composition may influence the mode of nucleation in cirrus clouds, and as such, chemical characteristics of the particles may potentially be important. The chemical properties will be the topic of a subsequent study.

Acknowledgements. We acknowledge the work by the flight department at DLR during the campaign. L. Bäcklin and N. Walberg were very helpful in the work with the instruments. We thank B. Noone for solving all kinds of problems and S. Wilhelm for excellent support during the INCA preparation in Oberpfaffenhofen. Helpful discussions with $\mathrm{P}$. Tunved about MATLAB related problems are also acknowledged. This work was supported by the European Union through the INCA project (Contract no. EVK2-CT-1999-00039).

\section{References}

Ackerman, A. S., Toon, O. B., Taylor, J. P., Johnson, D. W., Hobbs, P. V., and Ferek, R. J.: Effects of aerosols on cloud albedo: Evaluation of Twomey's parameterization of cloud susceptibility using measurements of ship tracks, J. Atmos. Sci., 57, 2684-2695, 2000
Baehr, J., Schlager, H., Ziereis, H., Stock, P., Velthoven, P., Busen, R., Ström, J., and Schumann, U.: Aircraft observations of $\mathrm{NO}, \mathrm{NO}_{\mathrm{y}}, \mathrm{CO}$, and $\mathrm{O}_{3}$ in the upper troposphere from $60^{\circ} \mathrm{N}-60^{\circ} \mathrm{S}-$ Interhemispheric differences and distributions over tropical South America, Geophys. Res. Lett., 30, 10.1029/2002GL016776, 2003.

Baumgardner, D., Dye, J. E., Gandrud, B. W., and Knollenberg, R. G.: Interpretation of measurements made by the forward scattering spectrometer probe (FSSP-300) during the Airborne Arctic Stratospheric Expedition, J. Geophys. Res., 97, 8035-8046, 1992.

Borrmann, S., Luo, B. P., and Mishchenko, M.: Application of the T-matrix method to the measurement of aspherical (ellipsoidal) particles with forward scattering optical particle counters, J. Aerosol Sci., 31, 789-799, 2000.

Chen, J. P. and Crutzen, J.: Solute effects on the evaporation of ice particles, J. Geophys. Res., 99, 18 847-18 859, 1994.

Cohard, J. M., Pinty, J. P., and Bedos, C.: Extending Twomey's analytical estimate of nucleated cloud droplet concentrations from CCN spectra, J. Atmos. Sci., 55, 3348-3357, 1998.

DeMott, P. J., Meyers, M. P., and Cotton, W. R.: Parameterization and Impact of Ice Initiation Processes Relevant to NumericalModel Simulations of Cirrus Clouds, J. Atmos. Sci., 51, 77-90, 1994.

Gayet, J.-F., Auriol, F., Minikin, A., Ström, J., Seifert, M., Krejci, R., Petzold, A., Febvre, G., and Schumannn, U.: Quantitative measurement of the microphysical and optical properties of cirrus clouds with four different in situ probes: Evidence of small ice crystals, Geophys. Res. Lett., 29, 2230, 2002.

Gerbig, C., Kley, D., Volz-Thomas, A., Kent, J., Dewey, K., and McKenna, D. S.: Fast response resonance fluorescence CO measurements aboard the C-130: Instrument characterization and measurements made during North Atlantic Regional Experiment 1993, J. Geophys. Res., 101, 29 229-29 238, 1996.

Haywood, J. and Boucher, O.: Estimates of the direct and indirect radiative forcing due to tropospheric aerosols: A review, Reviews of Geophysics, 38, 513-543, 2000.

Intergovermental Panel on Climate Change (IPCC), Climate Change 2001: The Science of Climate Change, Third Assessment Report, edited by Houghton, J. T., et al., Cambridge Univ. Press, Cambridge, UK, 944pp, 2001.

Jensen, E. J. and Toon, O. B.: Ice Nucleation in the Upper Troposphere - Sensitivity to Aerosol Number Density, Temperature and Cooling Rate, Geophys. Res. Lett., 21, 2019-2022, 1994.

Jensen, E. J. and Toon, O. B.: The potential impact of soot particles from aircraft exhaust on cirrus clouds, Geophys. Res. Lett., 24, 249-252, 1997.

Kärcher, B. and Lohmann, U.: A Parameterization of cirrus cloud formation: Homogeneous freezing including effects of aerosol size, J. Geophys. Res., 107, art. no.-4698, 2002.

Kärcher, B. and Lohmann, U.: A parameterization of cirrus cloud formation: Heterogeneous freezing, J. Geophys. Res., 108, 10.1029/JD2002003220, 2003.

Kärcher, B. and Ström, J.: The roles of dynamical variability and aerosols in cirrus cloud formation, Atmos. Chem. Phys., 3 ,823838, 2003.

Kristensson, A., Gayet, J. F., Strom, J., and Auriol, F.: In situ observations of a reduction in effective crystal diameter in cirrus clouds near flight corridors, Geophys. Res. Lett., 27, 681-684, 
2000.

Lohmann, U. and Kärcher, B.: First interactive simulations of cirrus clouds formed by homogeneous freezing in the ECHAM general circulation model, J. Geophys. Res., 107, art. no.-4105, 2002.

Minikin, A., Petzold, A., Ström, J., Krejci, R., Seifert, M., Velthoven, P., Schlager, M., and Schumann, U.: Aircraft observations of the upper tropospheric fine particle load in the northern and southern hemispheres at midlatitudes, Geophys. Res. Lett., 30, doi: 10.1029/2002GL016458, 2003.

Noone, K. J., Johnson, D. W., Taylor, J. P., Ferek, R. J., Garrett, T., Hobbs, P. V., Durkee, P. A., Nielsen, K., Ostrom, E., O’Dowd, C., Smith, M. H., Russell, L. M., Flagan, R. C., Seinfeld, J. H., De Bock, L., Van Grieken, R. E., Hudson, J. G., Brooks, I., Gasparovic, R. F., and Pockalny, R. A.: A case study of ship track formation in a polluted marine boundary layer, J. Atmos. Sci., 57, 2748-2764, 2000.

Ogren, J. A., Heintzenberg, J., and Charlson, R. J.: In-situ sampling of clouds with a droplet to aerosol converter, Geophys. Res. Lett., 12, 121-203, 1985.

Ovarlez, J., van Velthoven, P., Sachse, G., Vay, S., Schlager, H., and Ovarlez, H.: Comparison of water vapor measurements from POLINAT 2 with ECMWF analyses in high-humidity conditions, J. Geophys. Res., 105, 3737-3744, 2000.

Pruppacher, H. R. and Klett, J. D.: Microphysics of clouds and precipitation, Kluwer Academic Publishers, Dordrecht, the Netherlands, pp. 191-209, 1997.

Sassen, K., Starr, D. O., and Uttal, T.: Mesoscale and Microscale Structure of Cirrus Clouds - 3 Case Studies, J. Atmos. Sci., 46, 371-396, 1989.
Schlager, H., Konopka, P., Schulte, P., Schumann, U., Ziereis, H., Arnold, F., Klemm, M., Hagen, D. E., Whitefield, P. D., and Ovarlez, J.: In situ observations of air traffic emission signatures in the North Atlantic flight corridor, J. Geophys. Res., 102, 10739-10750, 1997.

Schröder, F. and Ström, J.: Aircraft measurements of sub micrometer aerosol particles $(>7 \mathrm{~nm})$ in the midlatitude free troposphere and tropopause region, Atmos. Res., 44, 333-356, 1997.

Seifert, M., Ström, J., Krejci, R., Minikin, A., Petzold, A., Gayet, J.F., Schumann, U., and Ovarlez, J.: In situ observations of aerosol particles remaining from evaporated cirrus crystals: Comparing clean and polluted air masses, Atmos. Chem. Phys., 3, 1-13, 2003.

Ström, J., Okada, K., and Heintzenberg, J.: On the State of Mixing of Particles Due to Brownian Coagulation, J. Aerosol. Sci., 23, 467-480, 1992.

Ström, J. and Ohlsson, S.: In situ measurements of enhanced crystal number densities in cirrus clouds caused by aircraft exhaust, J. Geophys. Res., 103, 11355-11 361, 1998.

Ström, J., Seifert, M., Krejci, R., Minikin, A., Petzold, A., Auriol, F., Ovarlez, J., and Schumann, U.: A statistical approach to a Lagrangian perspective of cirrus evolution based on data from the INCA experiments in the northern and southern hemisphere midlatitudes. Procceedings of the AMS conference on Cloud Physics, Odgen Utah, p. 6.3, 2002.

Twomey, S.: Pollution and the planetary albedo, Atmos. Environ., 8, 1251-1256, 1974. 